\title{
Epidemiology, clinical characteristics, resistance, and treatment of infections by Candida auris
}

\author{
Andrea Cortegiani ${ }^{1 *}$ (D), Giovanni Misseri ${ }^{1}$, Teresa Fasciana², Anna Giammanco ${ }^{2}$, Antonino Giarratano ${ }^{1}$ and \\ Anuradha Chowdhary ${ }^{3}$
}

\begin{abstract}
Candida spp. infections are a major cause of morbidity and mortality in critically ill patients. Candida auris is an emerging multi-drug-resistant fungus that is rapidly spreading worldwide. Since the first reports in 2009, many isolates across five continents have been identified as agents of hospital-associated infections. Independent and simultaneous outbreaks of $C$. auris are becoming a major concern for healthcare and scientific community. Moreover, laboratory misidentification and multi-drug-resistant profiles, rarely observed for other non-albicans Candida species, result in difficult eradication and frequent therapeutic failures of $C$. auris infections. The aim of this review was to provide an updated and comprehensive report of the global spread of $C$. auris, focusing on clinical and microbiological characteristics, mechanisms of virulence and antifungal resistance, and efficacy of available control, preventive, and therapeutic strategies.
\end{abstract}

Keywords: C. auris, Candida, Candidemia, Invasive fungal infection, Antimicrobial resistance, Antifungal resistance

\section{Introduction}

Candida spp. infections are a major cause of morbidity and mortality in critically ill patients [1-3]. Yeasts of genus Candida are associated with a wide range of different clinical manifestations, including bloodstream infections (BSIs), intra-abdominal candidiasis, deep-seated candidiasis, and superficial infections $[1,4,5]$. Infections caused by Candida spp. have progressively increased over the last decades, and this phenomenon is mainly associated with the increasing rate of invasive procedures, the extensive use of broad-spectrum antimicrobials, and the more frequent immunocompromised status of critically ill patients [6-8]. Although Candida albicans still remains the main agent of hospital-acquired fungal infection, several species of non-albicans Candida namely C. tropicalis, C. glabrata, C. parapsilosis, and C. krusei account for increasing incidence of invasive infections with high rates of

\footnotetext{
* Correspondence: andrea.cortegiani@unipa.it

${ }^{1}$ Department of Surgical, Oncological and Oral Science (Di.Chir.On.S.). Section of Anesthesia, Analgesia, Intensive Care and Emergency. Policlinico Paolo Giaccone. University of Palermo, Italy, University of Palermo, Via del vespro 129, 90127 Palermo, Italy

Full list of author information is available at the end of the article
}

therapeutic failure, mainly related to echinocandins and azoles resistance [9-11]. Current increase in antifungal drug resistance is not only linked to the acquired mechanism following administration of antifungal agents but intrinsic resistance to several classes of antimicrobials among different non-albicans species has also been recorded [12].

C. auris is an emerging multi-drug-resistant fungus that is rapidly spreading worldwide. Since the first reports in 2009, many isolates have been identified across five continents as agents of hospital-associated infections $[11,13,14]$. Reported cases are characterized by high overall mortality $[15,16]$ and high rate of antifungal resistance [17]. Of note, most reported infections involved critically ill patients $[15,18]$. Moreover, difficulty in microbiological identification $[19,20]$, high virulence [21-23], multi-drug resistance profile [24, 25], and rapid global spread with several reported outbreaks ([11, 26, 27]; (https://www.cdc.gov/fungal/diseases/candidiasis/ tracking-c-auris.html); [28]) lead the healthcare and scientific communities to consider $C$. auris as one of the most serious emerging pathogen that critical care physicians should be aware of. 
The aim of this review is to provide an updated report of the global spread of $C$. auris focusing on clinical and microbiological characteristics, mechanisms of virulence and antifungal resistance, and efficacy of available control, preventive, and therapeutic strategies.

\section{Main text}

\section{Systematic review}

For the purpose of this review, we performed a systematic review of the literature using "Candida" AND "auris" as keywords. We searched the PubMed, Scopus, and Web of Science. We excluded articles in languages other than English. Two authors (A. C. and G. M.) independently performed the search. Differences in selections were solved by consensus, with the help of the third author (T. F.). We included peer-review articles and meeting abstracts, concerning epidemiology, clinical manifestations and risk factors, virulence, genotypic characteristics, and therapeutic management. Concerning clinical cases, we included all cases of isolation of $C$. auris in humans reported in literature. Cases were defined as patients in whom C. auris was isolated, and this definition includes both superficial and deep-seated infections. We also checked references of relevant articles to find potential articles not retrieved by the databases search. After excluding not relevant articles and duplicates, we included 131 relevant articles published from 2009 to 30 May 2018. Articles retrieved were further categorized as shown in the flow diagram, following PRISMA guidelines (Additional file 1).

\section{Microbiological characteristics of C. auris}

On Sabouraud's agar, C. auris produces smooth and white cream-colored colonies, which are germ tube test negative. On CHROMagar Candida medium, C. auris produces colonies that may appear pale to dark pink, or rarely beige. The yeast $C$. auris is able to grow at $42{ }^{\circ} \mathrm{C}$, and this characteristic helps differentiate $C$. auris from C. haemulonii, which does not grow at these temperatures [19]. The microscopic morphology of C. auris cells appears to be oval without pseudohyphae formation. However, C. auris might exhibit multiple morphological phenotypes under different cultures conditions, including round-to-ovoid, elongated, and pseudohyphal-like forms. For instance, high concentrations of sodium chloride induce the formation of a pseudohyphal-like form [29]. Cycloheximide $0.1 \%$ and $0.01 \%$ inhibits its growth [30]. The phenotypic, chemotaxonomic, and phylogenetic characteristics (Fig. 1) have therefore clearly suggested that it was a new species affiliated to the genus Candida (anamorphic) and therefore to the class of Ascomycetes even if the perfect form is not known (teleomorphic). Whole genome phylogeny of $C$. auris, C. haemulonii, C. duobushaemulonii, and C. pseudohaemulonii showed that they represent a single clade, confirming the close relationship of these species [31]. Due to the close genetic relatedness with $C$. haemulonii complex, $C$. auris is often commonly misidentified as $C$. haemulonii in routine diagnostic laboratories using biochemical methods. In fact, commercially available biochemical-based tests, including API AUX 20C, VITEK-2 YST, BD Phoenix, and MicroScan, misidentify C. auris as a wide range of Candida species and other genera. Misidentifications yielding C. famata, C. sake, Rhodotorula glutinis, Rhodotorula mucilaginosa, Saccharomyces, C. catenulate, C. lusitaniae, C. guilliermondii, and C. parapsilosis have been reported $[19,20,26]$. Recently, BioMerieux has updated the database [32, 33] and inclusion of $C$. auris spectra in the VITEK-2 system yields to its correct identification. Matrix-assisted laser desorption/ionization time-of-flight (MALDI-TOF) mass spectrometry can reliably differentiate $C$. auris from other Candida species, provided C. auris spectrum is included in the reference database and by selecting appropriate extraction method [34,35]. The development of specific PCR assays for C. auris and for $C$. auris-related species using cultured colonies seems promising for its rapid and accurate identification, particularly in outbreak settings [36, 37]. Molecular identification of $C$. auris can be performed by sequencing various genetic loci (including $D 1 / D 2, R P B 1, R P B 2$, and internal transcribed spacer ITS1, ITS2), but it is not routinely used [38, 39].

\section{Epidemiology trends and world outbreaks}

The real prevalence and the epidemiology of $C$. auris still remain uncertain. One of the causes may be the underestimation of its isolation due to the limited accuracy of available conventional diagnostic tools [40]. With the purpose to investigate whether $C$. auris emerged in recent times or had been misidentified in the past, an extensive investigation was conducted within the pool of uncommon Candida spp. included in the SENTRY global fungal collection (15,271 isolates of Candida spp. from four continents) [41]. This study identified a single C. auris isolate from Pakistan dating back to 2008, which had not been previously recognized [41]. In 2011, Lee et al. reported the first three cases of bloodstream fungemia caused by $C$. auris highlighting antifungal resistance and the ability to cause invasive infections [42]. One of these cases was incidentally recognized by molecular identification of a microbiological sample obtained in 1996 as invasive fungal infection isolate. To our knowledge, there are no other unidentified $C$. auris strains prior to 1996.

The first "named" description of $C$. auris as a new emergent pathogen has been reported in 2009 by Satoh et al. [13]. The authors reported a single isolate from the discharge of the external ear canal of a 70-year-old 


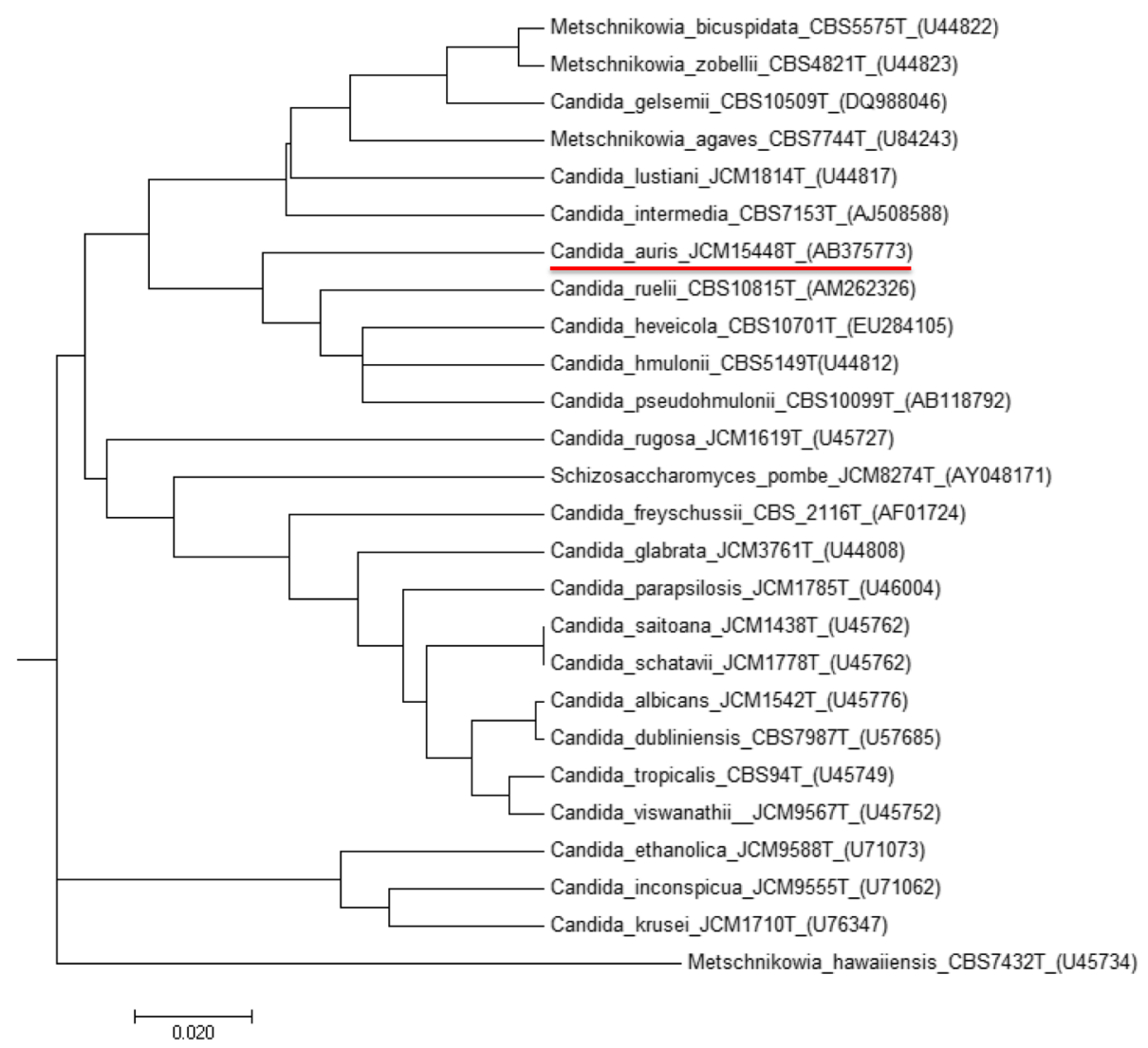

Fig. 1 Phylogenetic tree obtained by neighbor-joining analysis of the D1-D2 region of genes encoding Candida auris 26S rRNA and correlated species

inpatient at Tokyo Metropolitan Geriatric Hospital (Tokyo, Japan). Phenotypic, chemotaxonomic, and phylogenetic analyses indicated an affiliation to Candida genus, with a close relation to other unusual species [13] such as C. haemulonii and C. pseudohaemulonii. Later, in South Korea [14], 15 patients affected by chronic otitis media were identified to be infected by unusual and clonally related yeast isolates of $C$. auris confirmed by genomic sequencing [43]. Since the first isolation, $C$. auris infections have been reported from many countries, including India [15, 24, 38, 44], Pakistan [41], South Korea [42], Malaysia [45], South Africa [46], Oman [47, 48], Kenya [49], Kuwait [50], Israel [51], United Arab Emirates [52], Saudi Arabia [53], China [54], Colombia [55-57], Venezuela [58], the United States (US) ((https://www.cdc.gov/fungal/diseases/candidiasis/tracking-c-auris.html); [59-61]), Russia [62], Canada [63], Panama [64, 65], the United Kingdom (UK) [66], and continental Europe [28, 67-70]. Figure 2 shows C. auris reported isolations in chronological order. Figure 3 shows the worldwide distribution.

Europe's burden of $C$. auris outbreaks appears to be increasing, although the epidemiological profile is not completely defined [28]. Recently, the ECDC published a survey on reported cases of $C$. auris and laboratory capacity in Europe, with the purpose to implement surveillance and to control its further spread [28]. Six hundred and twenty cases of $C$. auris were reported in a period from 2013 to 2017, with two countries experiencing four hospital outbreaks. Sporadic cases have been identified since 2013 from different patients throughout England. The first outbreak of $C$. auris in Europe occurred in a London cardio-thoracic center between April 2015 and July 2016; 50 cases were identified, with ability for rapid colonization and transmissibility within the healthcare setting, leading to a serious and prolonged outbreak [66]. The first $C$. auris invasive infection in continental Europe occurred in Spain, where four patients admitted to the surgical intensive care unit of Valencia $\mathrm{La} \mathrm{Fe}$ University and Polytechnic Hospital (Valencia, Spain) between April and June 2016 were diagnosed with deep-seated infection caused by this "super-fungus" [67] Despite efforts in limiting diffusion of this pathogen, new colonization cases have continued to appear until now, with a tendency to acquire an endemic pattern. During the study period from April 2016 to January 2017, 140 patients were colonized by $C$. auris and 41 patients underwent candidemia episodes, with 5 patients 


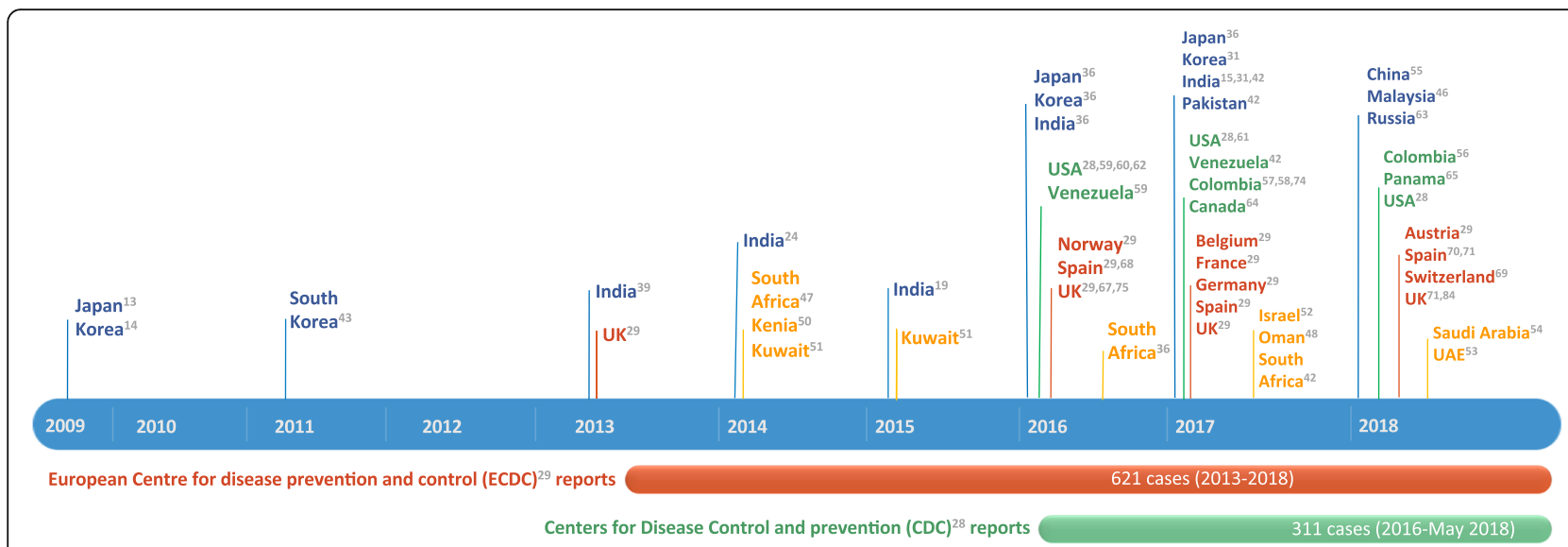

Fig. 2 Timeline chart of $C$. auris reported cases. The reports from the European Centre for Diseases Prevention and Control (ECDC) and the Centers for Disease Control and Prevention are ongoing

developing septic metastatic complications. This is the largest ongoing European clonal outbreak [69], involving a different strain from those previously reported, as demonstrated by genotype analysis.

Chowdhary et al. in 2013 were the first to report an outbreak of $C$. auris infection in India, identifying 12 patients with positive microbiological clinical samples collected between 2009 and 2012 [38]. Since then, there has been a progressive increase in the number of clinical cases reported. The high prevalence of invasive infections due to $C$. auris has become a great concern in India, as inter- and intra-hospital spreading of this multi-resistant pathogen has been demonstrated [15]. Public Indian institutions are characterized by higher prevalence of C. auris isolation than private hospitals, possibly connected to overcrowding and compromised infection control measures [15], with $C$. auris prevalence ranging from 5 to $30 \%$ of all candidemia cases in certain institutions [15, 24, 38, 44]. Recently, C. auris was found to be the second most prevalent species causing candidemia in a tertiary care trauma center in Delhi, India, warranting more effective infection control practices to prevent its spread [43]. Moreover, outbreaks of candidemia in Pakistan could be related to the interregional spread of the pathogen, as demonstrated by genomic sequencing of Indian and Pakistani isolates [41].

In US, the Center of Disease Control and Prevention (CDC) issued a clinical alert in June 2016 informing

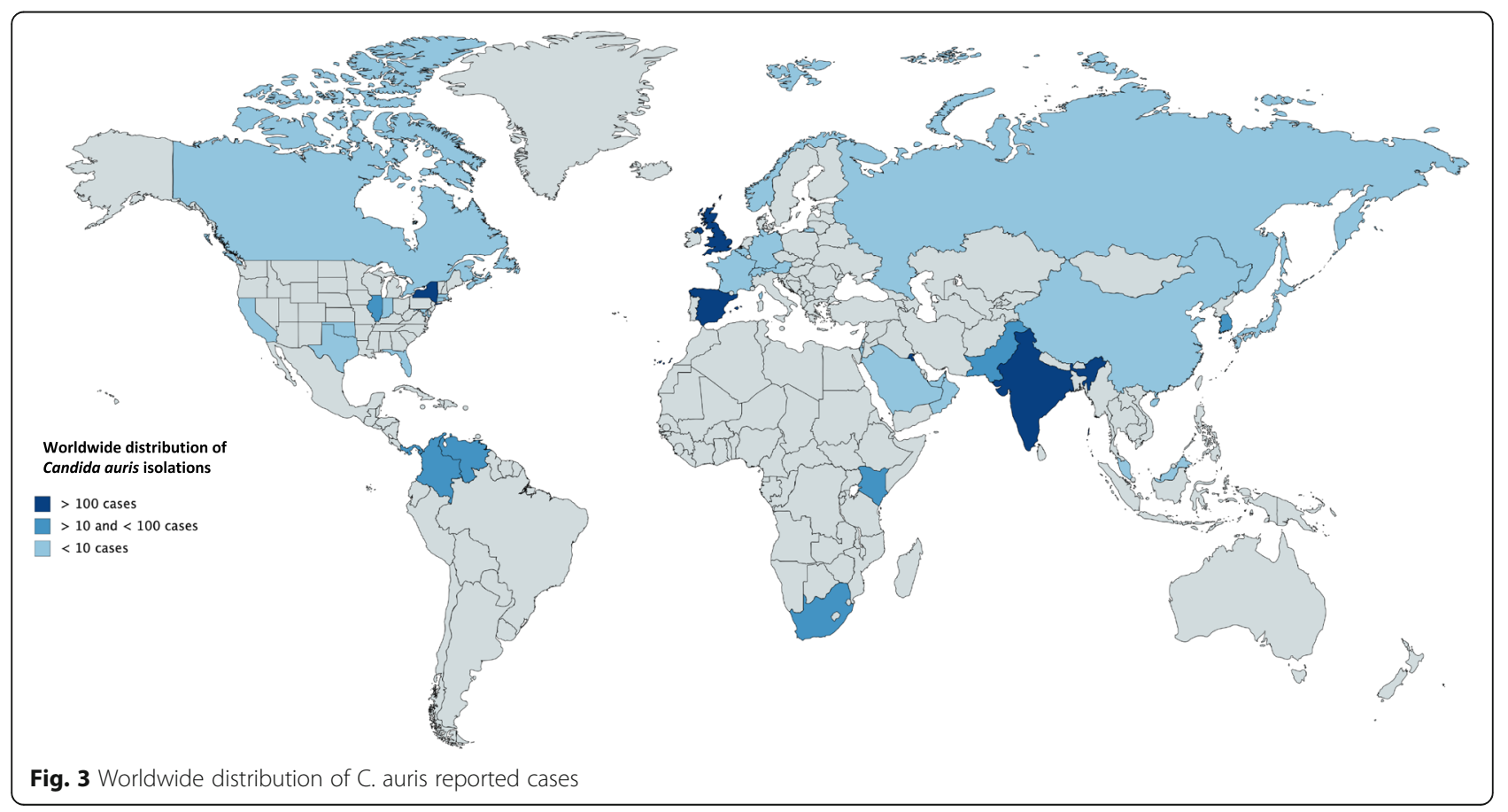


clinicians, laboratories, infection control practitioners, and public health authorities about $C$. auris. It requested that all cases be adequately reported to authorities and to the CDC [71, 72]. This report describes the first seven US cases of $C$. auris infection occurring during May 2013 and August 2016. Six of seven cases were identified through retrospective review of microbiology records from reporting hospitals and reference laboratories [60]. As of May 2018, CDC recorded 311 confirmed and 29 probable cases of $C$. auris infection. Most C. auris isolates in the US have been detected in the New York City area and New Jersey (https://www.cdc.gov/fungal/disease s/candidiasis/tracking-c-auris.html). Available epidemiological information suggests that most strains of $C$. auris isolated in the United States were introduced from abroad. Isolates from Illinois and New York were of the same clade as those from South America and South Asia respectively [61]. However, it is not possible to exclude that most of these cases were acquired in the US following local transmission in healthcare settings [60].

Although imported cases have been demonstrated as in US healthcare outbreaks, one of the major questions regarding C. auris spread is whether it emerged independently in different countries or if a single outbreak spread from an original source [40]. Using WGS (whole genome sequencing) and SNP (single-nucleotide polymorphism) analysis together with epidemiological observation $[25,41]$, it was possible to demonstrate an independent and simultaneous emergence of different $C$. auris clonal populations on different geographical areas. Specifically, it was possible to identify the emergence of four different clades (East and South Asian, African, South American) in as many different regions. Transmission within the healthcare setting is suggested by the clonal relatedness of isolates in different institutions [24, 38, 40, 67].

Different reports have been published from South America. The first outbreak was reported in Venezuela between March 2012 and July 2013 [58]. All the isolates were initially identified as C. haemulonii. However, isolation of $C$. auris was later confirmed by genome sequencing. The Venezuelan outbreak resulted in $C$. auris being the sixth most common cause of candidemia in the involved institution. In Colombia, sporadic cases have been reported since 2012 [55-57]. Interestingly, an outbreak was reported in a pediatric intensive care unit in 2016, where five cases of invasive infections were identified. Shortly after, nine cases have been isolated in Panama, where patterns of resistance detected by both microdilution method were similar to those observed among C. auris isolates in Colombia [65].

In Africa, the first identification of sporadic cases and outbreaks were in South Africa and Kenya. The first four South African cases were isolated in 2012-2013 [46]. Other 10 isolates have been detected, demonstrating a close relation but phylogenetically distinct from Pakistan, India, and Venezuela [41]. Instead, according to other studies, isolates from South Africa had sequence similarity with those from UK [73]. C. auris has been considered as the most common pathogen responsible for candidemias in a reference hospital in Kenya, accounting for 45 (38\%) episodes over a nearly 3-year period [49].

Only a single report of $C$. auris candidaemia has been published to date in Israel [17]. Collected strains were phylogenetically different from those from East Asia, Africa, and the Middle East, indicating an independent emergence of the pathogen. Infections have been reported in different Gulf States, including Kuwait [50], Oman [47, 48], and United Arab Emirates [52]. Recently, the first three cases in Saudi Arabia have been reported [53].

\section{Clinical characteristics, risk factors, and outcome}

In most cases, clinical presentation is non-specific and it is often difficult to differentiate between other types of systemic infections. Most of the reported cases in the last 5 years were isolated from blood and other deep-seated sites of infection (including invasive devices and catheters tips) [16]. Different clinical conditions including bloodstream infections, urinary tract infection, otitis, surgical wound infections, skin abscesses (related to insertion of the catheter), myocarditis, meningitis, bone infections, and wound infections have been related to $C$. auris $[15,18]$. However, isolations from non-sterile body sites such as lungs, urinary tract, skin and soft tissue, and genital apparatus may more likely represent colonization rather than infections $[18,74]$. As for other Candida spp., the presence of signs and symptoms of infections of the site where $C$. auris has been isolated from can help to differentiate between simple colonization and infection [4]. It is important to identify C. auris even from a non-sterile body site because colonization poses the risk of transmission, which requires implementation of infection control precautions [71, 72].

To investigate risk factors associated to $C$. auris infections, Rudramurthy et al. conducted a subgroup analysis and comparison of the clinical manifestations of $C$. auris and non-auris cases in 27 Indian ICUs [15]. In accordance with previous studies, risk factors were not different from those associated with invasive infection due to other Candida spp. [22], including prior or continuous exposure to broad-spectrum antibiotics and antifungal agents, diabetes mellitus, abdominal and vascular surgery, presence of central venous catheters, urinary catheterization, post-operative drain placement, chronic kidney disease, chemotherapy, blood transfusions, hemodialysis, total parenteral nutrition, immunosuppressive state [75] and neutropenia [45], and length of ICU stay $[15,18,76]$. The incidence of $C$. auris is 
significantly higher in patients with primary or acquired altered immune response, secondary to therapeutic management of hematologic malignancies, bone marrow transplantation, and other condition requiring immunosuppressive agents [60]. Interestingly, Azar et al. reported the first case of donor-derived transmission of $C$. auris in a lung transplant patient [75], highlighting several implications on microbiological surveillance before transplants.

The crude in-hospital mortality rate for C. auris candidemia is estimated to range from 30 to $72 \%[16,26,41$, $44,69]$. Available data suggest that the vast majority of infections affects adults, with a propensity for critically ill patients in intensive care unit (ICU) settings. Pediatric patients have only been reported in Asia and South America [77]. A better outcome was seen in this population $[42,58,77]$.

\section{Infection prevention and control}

The progressive increase of outbreaks and sporadic cases of $C$. auris infection emphasize the need for adequate prevention measures. According to reports of recent outbreaks, colonization is difficult to eradicate and it tends to persist for months $[66,69]$. Prevention of outbreaks has to be based on the early recognition of sporadical cases, identification of reservoirs and prompt notification. Guidance has been released by international organizations such as Public Health England (PHE-UK) [78], the CDC [79], the ECDC [70], and the Center for Opportunistic Tropical and Hospital Infections (COTHI-South Africa) [80], with recommendations regarding the isolation of patients, contact precautions, and cleaning of equipment and environments in contact with affected patients. Prevention and infection control policies are empirical and mainly based on indications formulated for containment strategies for other multi-drug-resistant pathogens. Table 1 summarizes recommendations by the CDC and the ECDC for prevention and control of $C$. auris transmission.

Although the exact mode of transmission has to be identified, early evidence suggests that $C$. auris spread is mainly related to exposure to contaminated facilities and transmission from healthcare personnel. Persistent outbreaks have been associated with hand transmission and contamination of surfaces $[61,66,81,82]$. However, the

Table 1 Key points for C. auris prevention and control by the European Centre for Diseases Prevention and Control (ECDC) and Centers for Disease Control and Prevention (CDC)

ECDC CDC

Correct identification (MALDI-TOF; DNA sequencing of the D1/D2 domain);

Clinicians and microbiologists alertness:

Notification and retrospective case-finding

Good standard infection control measures (including environmental cleaning, reprocessing of medical devices and patient isolation) and prompt notification

Early identification of carriers by using active surveillance cultures (sites considered for sampling include nose/throat, axilla, groin, rectum, insertion sites of venous catheters; clinical samples such as urine, feces, wound drain fluid, and respiratory specimens)

Establish the source of the outbreak (epidemiological investigation, cross-sectional patient screening and environmental sampling); prevention of inter-hospital and cross-border transmission

Enhanced control measures to contain outbreaks (such as contact precautions, single room isolation or patient cohorting, and dedicated nursing staff for colonized or infected patients)

Education and practice audits (for healthcare workers and contacts)
Correct identification (MALDI-TOF; molecular methods) Confirmed isolates of $C$. auris should be reported to local and state public health officials and to CDC

Infection control measures:

- Placing the patient with C. auris in a single-patient room and using contact precautions

- Emphasizing adherence to hand hygiene

- Cleaning and disinfecting the patient care environment (daily and terminal cleaning) with recommended products

- Screening contacts of newly identified case patients to identify C. auris colonization

Screening should be performed to identify colonization among potentially epidemiologically linked patients, including:

- Current roommates

- Roommates at the current or other facilities in the prior month (even if they have been discharged from the facility)

Screening for $C$. auris should be done using a composite swab of the patient's axilla and groin (sites of consistent colonization). Patients have also been found to be colonized with C. auris in nose, external ear canals, oropharynx, urine, wounds, and rectum.

All laboratories, especially laboratories serving healthcare facilities where cases of $C$. auris have been detected, should:

- Review past microbiology records to identify cases of confirmed or suspected C. auris

- Conduct prospective surveillance to identify C. auris cases in the future

- Consider screening close contacts of patients with $C$. auris for presence of colonization

Education of all healthcare personnel, including staff working with environmental cleaning services about C. auris and need for appropriate precautions;

Monitor adherence to infection control practices

Antibiotic and antifungal stewardship 
role of healthcare workers still remains difficult to determine. A recent study sampled patients and their contacts, healthcare workers, and environment in four hospitals in Colombia that had previously reported $C$. auris outbreaks, and found $C$. auris on different objects and facilities, such as bedrails, a bed hand-controller, a mobile phone, and floors. Interestingly, positive samples were collected from surfaces with infrequent patient contact but frequent healthcare workers contact (i.e., chairs, bed trays, and medical equipment), and from surfaces with little to no patient contact and infrequent healthcare workers contact (i.e., closet cabinets, door handles, alcohol gel dispensers) [83]. Thus, once C. auris is introduced in the hospital setting, environmental contamination evolves well beyond the patient bedside, resulting in recurrent cases of new colonizations.

C. auris is able to survive on a wide range of dry and moist surfaces, including plastic where the pathogen may reside for up to 14 days [84]. C. auris seems to be resistant to quaternary compounds disinfectants and cationic surface-active products. Disinfectants with sporicidal activity and hydrogen peroxide-based products are indicated to clean surfaces and healthcare facilities, resulting in highest reduction of $C$. auris colony-forming unit (CFU) [81, 85, 86]. Chlorine-based detergents, ultraviolet light, and hydrogen peroxide vapor demonstrated their efficacy in environmental decontamination procedures after patient discharge [61, 66, 87]. However, persistence of $C$. auris within the hospital environment despite disinfection procedures also suggests an involvement of the interaction between the pathogen and surfaces and the length of exposure to disinfectants [88].

In order to curb transmission, authorities recommend adherence to central and peripheral catheter care bundles, urinary catheter care bundle, and care of tracheostomy sites [78, 79]. If feasible, removal of central catheters or other invasive devices may resolve persistent candidemia and improve clinical outcome [58, 67]. Patients colonized or with proven or suspected C. auris infection should be kept in isolation under strict contact precautions until microbiological screening and diagnostic results are available [66]. Incoming patients from institutions where proven $C$. auris isolation has been determined should be screened [78]. Suggested screening sites are groin and axilla, urine, nose and throat, perineal and rectal swab or stool sample. Other high-risk sites may be of consideration, including wounds, cannula entry sites, endotracheal secretions, and drain fluids [70].

\section{C. auris virulence factors}

C. auris possesses virulence factors, such as germination, adherence, formation of biofilms, and production of phospholipases and proteinases [30]. Table 2 summarizes
Table 2 C. auris virulence and resistance factors

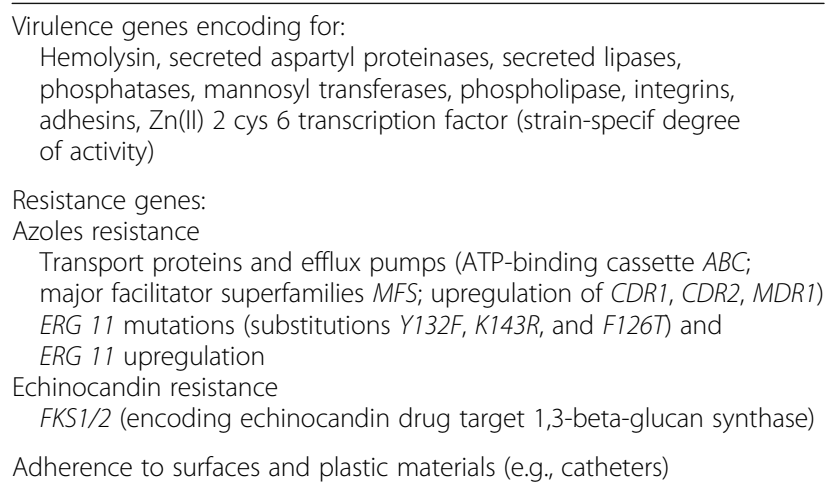

Biofilm formation

Cellular morphology (aggregating and non-aggregating forms)

Rudimentary pseudohyphae formation

C. auris virulence and resistance factors. Although compared to C. albicans, $C$. auris forms significantly reduced biofilms, nevertheless, it has the capacity to form adherent biofilm communities on a range of clinically important substrates. Larkin et al. studied 16 different $C$. auris isolates obtained from patients in Japan, India, South Korea, and Germany and characterized their morphology and virulence factors [30]. C. auris produces phospholipase and proteinase in a strain-dependent manner and exhibited a significantly reduced ability to adhere to catheter material as compared to that of $C$. albicans. Further, $C$. auris biofilms were mainly composed of yeast cells adhering to catheter material. In contrast, C. albicans showed a highly heterogeneous architecture of biofilms with yeast cells and hyphae embedded within the extracellular matrix [30]. Sherry et al. described the ability of $C$. auris to form antifungal-resistant biofilms, against all three main classes of antifungals [87]. These biofilms were shown to be resistant to chlorhexidine and hydrogen peroxide, displaying a less susceptible phenotype than C. albicans and C. glabrata [87, 89]. More recently, Kean et al. using a molecular approach investigated the genes that are important in causing the $C$. auris cells to be resistant within the biofilm [89]. Transcriptomic analysis of temporally developing $C$. auris biofilms was shown to exhibit phase- and antifungal class-dependent resistance profiles. Differential expression analysis demonstrated that 791 and 464 genes were upregulated in biofilm formation and planktonic cells, respectively, with a minimum twofold change. Notably, in the intermediate and mature stages of biofilm development, a number of genes encoding efflux pumps were upregulated, including ATP-binding cassette $(A B C)$ and major facilitator superfamily $(M F S)$ transporter suggesting efflux-mediated resistance in $C$. auris [89]. Previously, Ben-Ami et al. also reported significantly greater ABC-type efflux activity, as evidenced by Rhodamine 6G 
transport, among C. auris than C. glabrata isolates suggesting efflux-mediated intrinsic resistance of $C$. auris to azoles [17]. Virulence of $C$. auris and $C$. haemulonii has been recently compared with $C$. glabrata and C. albicans in an immunocompetent murine model of invasive infection. In this study, authors reported that virulence in $C$. auris appears to be similar to C. albicans and C. glabrata, suggesting that common gene sequences could play a pivotal role [23]. The whole genome data of the emerging multidrug resistant species and other related Candida revealed that $C$. auris shares some notable expansions of gene family described as related to virulence (including transporters and secreted lipases) in C. albicans and related pathogens [31]. The pathogenicity of $C$. auris compared to that of other common pathogenic yeast species in the invertebrate Galleria mellonella infection demonstrated strain-specific differences in the behavior of $C$. auris in $G$. mellonella, with the aggregate-forming isolates exhibiting significantly less pathogenicity than their non-aggregating counterparts. Importantly, the non-aggregating isolates exhibited pathogenicity comparable to that of C. albicans [29]. Finally, the ability of salt tolerance and cell clumping into large and difficult to disperse aggregates of $C$. auris can contribute to its resistance in the hospital environments. Despite the ability to possess the virulence factors, it is observed that the capacity of $C$. auris to express those is much weaker than that of other Candida spp., suggesting that this emerging species is not as virulent as the latter species [30, 87].

\section{C. auris profile of antifungal resistance and their mechanisms}

The ability of $C$. auris to develop resistance to multiple commonly used antifungal agents may be responsible for its high rates of mortality [76]. Antifungal susceptibility data published so far points out that some $C$. auris strains exhibit elevated minimum inhibitory concentration (MIC) for three major classes of antifungal drugs, i.e. azoles, polyenes, and echinocandins [41]. Table 3 shows C. auris MICs and tentative MICs breakpoint for the most common antifungal drugs.

C. auris is frequently resistant to fluconazole although isolates with low MICs against fluconazole (2$8 \mathrm{mg} / \mathrm{L}$ ) have also been recorded in India and Colombia $[57,83,90,91]$. Recently, reports have also documented high MICs to amphotericin B, voriconazole, and caspofungin. Antifungal susceptibility testing of 350 isolates of C. auris in 10 hospitals in India over an 8-year period showed that $90 \%$ of strains were resistant to fluconazole (MIC 32 to $\geq 64 \mathrm{mg} / \mathrm{L}$ ), $2 \%$ to echinocandins $(\mathrm{MIC} \geq 8 \mathrm{mg} / \mathrm{L}), 8 \%$ to amphotericin $\mathrm{B}(\mathrm{MIC} \geq 2 \mathrm{mg} / \mathrm{L}$ ) and $2.3 \%$ to voriconazole (MIC $16 \mathrm{mg} / \mathrm{L}$ ) [90]. In a recent report of $C$. auris candidemia in a tertiary care trauma center in Delhi, India, $45 \%$ of $C$. auris isolates exhibited low MICs of fluconazole [91]. Antifungal susceptibility testing of clinical blood isolates and isolates recovered from environmental and body swabs from hospitals in Colombia revealed that all isolates had low MICs to voriconazole, itraconazole, isavuconazole, and echinocandins [83]. The variable rates of azole resistance in different geographic regions suggest localized evolvement of resistance. Although, data underlying the molecular mechanisms related to resistance to common antifungal drug classes in C. auris is scarce, the following update is based on a few recent studies:

\section{a) Azole}

The resistance is mediated by point mutations in the lanosterol $14 \alpha$-demethylase (ERG11) gene. Substitutions $Y 132 F, K 143 R$, and $F 126 L$ in the gene were detected. Moreover, ERG11 gene expression can be increased fiveto sevenfold in the presence of fluconazole [90]. This gene, in some strains, can be present in an increased copy number, suggesting that increased copy number may be a mechanism of drug resistance in C. auris [91].

Table 3 Minimum inhibitory concentration (MIC) range and tentative MIC breakpoints of C. auris for most common antifungal drugs. Data retrieved by Centers of Disease Control and Prevention (CDC) website-https://www.cdc.gov/fungal/candida-auris/ recommendations.html

\begin{tabular}{lll}
\hline Drugs & MIC range $(\mathrm{mcg} / \mathrm{ml})$ & Tentative MIC breakpoints $(\mathrm{mcg} / \mathrm{ml})$ \\
\hline Triazoles & & $\geq 32$ \\
$\quad$ Fluconazole & 0.12 to $>64$ & $\mathrm{~N} / \mathrm{A}$ \\
$\quad$ Voriconazole (and other $2^{\circ}$ generation azoles) & $0.032-16$ & $\geq 2$ \\
Polyenes & & $\geq 4$ \\
$\quad$ Amphotericine B & $0.06-8$ & $\geq 2$ \\
Echinocandins & & $\geq 4$ \\
$\quad$ Anidulafungin & $0.015-16$ & $\geq 0$ \\
Caspofungin & $0.03-16$ & \\
Micafungin & $0.015-8$ & \\
\hline
\end{tabular}


Mutations in ERG11 gene associated with the development of fluconazole resistance in $C$. albicans have been detected in a global collection of 54 C. auris isolates including amino-acid substitutions specific with geographic clades: F126T with South Africa, Y132F with Venezuela, and $Y 132 F$ or K143F with India and Pakistan [41]. The ERG11 sequences of Indian $C$. auris showed amino acid substitutions at position $Y 132$ and $K 143$ for strains that were resistant to fluconazole, whereas genotypes without substitution at these positions were observed in isolates with low MICs of fluconazole (MIC 1-2 mg/L) [90]. These results suggest that these substitutions would give a phenotype of fluconazole resistance. Specific ERG11 substitutions in C. albicans, including F126T, Y132F, and $K 143 R$, are directly associated with resistance and have been shown to exhibit reduced susceptibilities to azoles upon heterologous expression in S. cerevisiae [92, 93].

Other mechanisms of azole resistance have been described in C. albicans, including upregulation of ERG11 and upregulation of drug efflux pumps (e.g., $C D R 1, C D R 2$, $M D R 1)$ due to gain of function mutations in transcription factors (e.g., TAC1, MRR1) that induce their expression [94]. The orthologs of transporters from the ATP-binding cassette $(A B C)$ and major facilitator superfamily (MFS) classes of efflux proteins have been reported in $C$. auris. Further, the overexpression of $C D R$ genes members of the $A B C$ family and MDR1 member of the MFS transporters has been recorded in $C$. auris isolates. Also, a single copy of the multidrug efflux pump MDR1 and 5-6 copy numbers of multidrug transporters such $C D R 1, S N Q 2$, and related genes have been identified in $C$. auris using WG sequence data [31], while the TAC1 transcription factor that regulates expression of $C D R 1$ and $C D R 2$ is present in two copies in C. auris [31].

\section{b) Echinocandins}

Main mechanisms of echinocandins resistance are mutations in the FKS1 gene encoding echinocandin drug target 1,3-beta-glucan synthase. FKS1 gene analysis using C. auris-specific $F K S$ primers in 38 Indian C. auris isolates showed that four $C$. auris isolates exhibited pan-echinocandin resistance (MICs $>8 \mathrm{mg} / \mathrm{L}$ ). All four resistant isolates had $S 639 F$ amino acid substitution equivalent to the mutation at position $S 645$ of the hot-spot 1 of FKS1, which is associated with resistance to echinocandins in C. albicans [90]. In contrast, in the remaining $34 \mathrm{C}$. auris isolates, wild-type phenotype was observed and the isolates exhibited low echinocandin MICs. Also, a single C. auris isolate resistant to both echinocandins and 5-flucytosine obtained from London Cardiothoracic outbreak was investigated for mutation analysis in the later study using WGS displayed SNP, causing a serine to tyrosine substitution $(S 652 Y)$ in the
FKS1 gene [95]. A recent study highlighted the challenges with the antifungal susceptibility testing of $C$. auris with caspofungin, as $F K S 1$ wildtype isolates exhibited an Eagle effect (also known as the paradoxical growth effect). Resistance caused by FKS1 S639F in C. auris was further confirmed in vivo in the mouse model of invasive candidiasis [96]. All isolates were susceptible at a human therapeutic dose of caspofungin, except for those exhibiting the $S 639 F$ aminoacid substitution. This result suggests that isolates demonstrating echinocandin resistance are characterized by mutations in FKS1 and that routine caspofungin antifungal susceptibility testing by broth microdilution method for $C$. auris isolates should be cautiously applied or even avoided [96]. However, micafungin is the most potent echinocandin in MIC testing and susceptibility testing with micafungin or FKS1 sequence analysis would be better indicators for detection of echinocandin resistance in C. auris [96].

\section{c) Amphotericin B}

The underlying mechanism of amphotericin $\mathrm{B}$ resistance has not been investigated so far in C. auris. A recent study by Escandon et al. aimed to describe the overall molecular epidemiology and resistances among Colombian C. auris isolates. The authors found that despite WSG revealed that isolates are genetically related throughout the country, higher resistance rates to amphotericin $\mathrm{B}$ were identified in northern regions if compared to central Colombia. Moreover, resistance to amphotericin B has been found to be significantly associated to four newly identified non-synonymous mutations [83]. Furthermore, reported data on susceptibility tests demonstrated that commercial systems (Vitek AST-YS07) could also detect false elevated MICs of amphotericin B. Thus, a cautious approach is recommended for laboratories to perform antifungal susceptibility testing for this yeast [19].

\section{Therapy: general concepts and new insights}

Echinocandins are the first-line therapy for C. auris infection, given resistance to azoles and amphotericine $\mathrm{B}$. As resistance to echinocandins has also been described, patients should undergo close follow-up and microbiological culture-based reassessment to detect therapeutic failure and eventual development of resistances. In cases of unresponsiveness to echinocandins, liposomial amphotericin $B$ (as single or combination therapy with an echinocandin) should be prescribed $[60,61,67,75]$ and consultation with an infectious diseases expert is recommended. Furthermore, MICs of azoles, such as itraconazole, posaconazole, and isavuconazole, are low and these drugs show good in vitro activity, possibly explained by the absence of previous exposure of yeast isolates to these agents, or because of the different structure of the azole-target-protein [41]. 
Drug associations have already been used with success $[60,67]$. Synergistic interactions may have a possible role, as demonstrated for micafungin and voriconazole association [23]. Considering the high prevalence and continuous spread of multi-drug resistant isolates of $C$. auris, there is the need to expand the classes of available antifungals. SCY-078 showed growth inhibition and anti-biofilm activity against $C$. auris isolates, with activity against echinocandin-resistant strains. Moreover, this drug is not affected by common mutations in protein targets and is orally bioavailable [97]. Recently, Basso et al. described the antifungal properties of $\theta$-defensins, 18-aminoacid macrocyclic peptides with potential applications for therapeutic treatment of systemic MDR infections, representing a template for the future development of new antifungals generation [98]. APX001 is a broad-spectrum antifungal agent for the treatment of invasive fungal infections, including species resistant to other antifungal drug classes, inhibiting an enzyme (Gwt1) part of the glycosylphosphatidylinositol (GPI) biosynthesis pathway [99]. Results of a study in a murine model of neutropenic disseminated candidiasis conducted by Zhao et al. may have potential relevance for clinical dose selection and breakpoints identification [100]. CD101 is a novel echinocandin with a prolonged half-life and an improved safety profile, allowing once weekly intravenous administration because of its enhanced pharmacokinetic properties [101]. In a recent study, Berkow et al. demonstrated an encouraging in vitro activity against most $C$. auris isolates, including strains resistant to other echinocandins [101].

\section{Conclusions}

Scientific community and clinicians are facing increasing incidence of antifungal resistance. Non-albicans Candida spp. infections are progressively emerging in hospitals and ICUs' settings. $C$. auris with high mortality rates, multi-drug resistance, environmental resilience, and horizontal transmission has become an issue in clinical practice. C. auris MDR strains may continue to emerge independently and simultaneously throughout the world in next few years. High level of knowledge and alertness by physicians and healthcare workers, especially in critical care settings, would help to control the spread and improve diagnostic and therapeutic strategies.

\section{Additional file}

Additional file 1: Flow diagram of the systematic search. (PDF $44 \mathrm{~kb}$ )

\section{Abbreviations}

ABC: ATP-binding cassette; AmB: Amphotericin B; BSI: Bloodstream infection; CDC: Center of Disease Control and Prevention; CFU: Colony-forming unit; COTHI-South Africa: Center for Opportunistic Tropical and Hospital Infections; ECDC: European Centre for Disease Prevention and Control;
GPI: Glycosylphosphatidylinositol; ICU: Intensive care unit; MALDI-TOF: Matrixassisted laser desorption/ionization time-of-flight; MDR: Multidrug resistant; MIC: Minimum inhibitory concentration; MRSA: Methicillin-resistant Staphylococcus aureus; PHE-UK: Public Health England; UK: United Kingdom; US: United States

\section{Acknowledgements \\ None. \\ Funding \\ None. \\ Availability of data and materials \\ Not applicable. \\ Authors' contributions \\ AC, GM, TF, A Giammanco, A Giarratano, and A Chowdhary conceived the content and wrote the manuscript. All authors read and approved the final version of the manuscript.}

Ethics approval and consent to participate

Not applicable.

Consent for publication

Not applicable.

\section{Competing interests}

The authors declare that they have no competing interests.

\section{Publisher's Note}

Springer Nature remains neutral with regard to jurisdictional claims in published maps and institutional affiliations.

\section{Author details}

'Department of Surgical, Oncological and Oral Science (Di.Chir.On.S.). Section of Anesthesia, Analgesia, Intensive Care and Emergency. Policlinico Paolo Giaccone. University of Palermo, Italy, University of Palermo, Via del vespro 129, 90127 Palermo, Italy. ${ }^{2}$ Department of Sciences for Health Promotion and Mother and Child Care, University of Palermo, Palermo, Italy. ${ }^{3}$ Department of Medical Mycology, Vallabhbhai Patel Chest Institute, University of Delhi, Delhi, India.

Received: 4 September 2018 Accepted: 15 October 2018

Published online: 29 October 2018

References

1. Kullberg BJ, Arendrup MC. Invasive candidiasis. N Engl J Med. 2015;373: 1445-56.

2. Cortegiani A, Russotto V, Maggiore A, Attanasio M, Naro AR, Raineri SM, et al. Antifungal agents for preventing fungal infections in non-neutropenic critically ill patients. Cochrane Database Syst Rev. 2016;1:CD004920.

3. Cortegiani A, Russotto V, Giarratano A. Associations of antifungal treatments with prevention of fungal infection in critically ill patients without neutropenia. JAMA. 2017;317:311-2.

4. Pappas PG, Kauffman CA, Andes DR, Clancy CJ, Marr KA, Ostrosky-Zeichner $L$, et al. Clinical practice guideline for the management of candidiasis: 2016 update by the Infectious Diseases Society of America. Clin Infect Dis. 2016; 62:e1-50.

5. Cortegiani A, Russotto V, Raineri SM, Gregoretti C, De Rosa FG, Giarratano A Untargeted antifungal treatment strategies for invasive candidiasis in nonneutropenic critically ill patients: current evidence and insights. Curr Fung Infect Rep. 2017;11:84-91.

6. Kett DH, Azoulay E, Echeverria PM, Vincent J-L. Candida bloodstream infections in intensive care units: analysis of the extended prevalence of infection in intensive care unit study. Crit Care Med. 2011;39:665-70.

7. Calandra T, Roberts JA, Antonelli M, Bassetti M, Vincent J-L. Diagnosis and management of invasive candidiasis in the ICU: an updated approach to an old enemy. Crit Care. 2016;20:125.

8. Bassetti M, Righi E, Ansaldi F, Merelli M, Scarparo C, Antonelli M, et al. A multicenter multinational study of abdominal candidiasis: epidemiology, outcomes and predictors of mortality. Intensive Care Med. 2015;41:1601-10. 
9. Cortegiani A, Misseri G, Chowdhary A. What's new on emerging resistant Candida species. Intensive Care Med. 2018 Online First doi: https://doi.org/ 10.1007/s00134-018-5363-X.

10. Lepak AJ, Zhao M, Berkow EL, Lockhart SR, Andes DR. Pharmacodynamic optimization for treatment of invasive Candida auris infection. Antimicrob Agents Chemother. 2017:61:e00791-17.

11. Chowdhary A, Sharma C, Meis JF. Candida auris: a rapidly emerging cause of hospital-acquired multidrug-resistant fungal infections globally. PLoS Pathog. 2017;13:e1006290.

12. Alexander $B D$, Johnson MD, Pfeiffer $C D$, Jimenez-Ortigosa C, Catania J, Booker $\mathrm{R}$, et al. Increasing echinocandin resistance in Candida glabrata: clinical failure correlates with presence of FKS mutations and elevated minimum inhibitory concentrations. Clin Infect Dis. 2013;56:1724-32.

13. Satoh K, Makimura K, Hasumi Y, Nishiyama Y, Uchida K, Yamaguchi H. Candida auris sp. nov., a novel ascomycetous yeast isolated from the external ear canal of an inpatient in a Japanese hospital. Microbiol Immunol. 2009:53:41-4.

14. Kim M-N, Shin JH, Sung H, Lee K, Kim E-C, Ryoo N, et al. Candida haemulonii and closely related species at 5 university hospitals in Korea: identification, antifungal susceptibility, and clinical features. Clin Infect Dis. 2009:48:e57-61.

15. Rudramurthy SM, Chakrabarti A, Paul RA, Sood P, Kaur H, Capoor MR, et al. Candida auris candidaemia in Indian ICUs: analysis of risk factors. J Antimicrob Chemother. 2017;72:1794-801.

16. Osei SJ. Candida auris: a systematic review and meta-analysis of current updates on an emerging multidrug-resistant pathogen. MicrobiologyOpen. 2018. https://doi.org/10.1002/mbo3.578.

17. Ben-Ami R, Berman J, Novikov A, Bash E, Shachor-Meyouhas Y, Zakin S, et al. Multidrug-resistant Candida haemulonii and C. auris, Tel Aviv, Israel. Emerg Infect Dis. 2017;23:195-203.

18. Chowdhary A, Voss A, Meis JF. Multidrug-resistant Candida auris: "new kid on the block" in hospital-associated infections? J Hosp Infect. 2016; 94:209-12.

19. Kathuria S, Singh PK, Sharma C, Prakash A, Masih A, Kumar A, et al. Multidrug-resistant Candida auris misidentified as Candida haemulonii: characterization by matrix-assisted laser desorption ionization-time of flight mass spectrometry and DNA sequencing and its antifungal susceptibility profile variability by Vitek 2, CLSI broth microdilution, and Etest method. J Clin Microbiol. 2015;53:1823-30

20. Kim T-H, Kweon OJ, Kim HR, Lee M-K. Identification of uncommon Candida species using commercial identification systems. J Microbiol Biotechnol. 2016:26:2206-13.

21. Finn T, Novikov A, Zakin S, Bash E, Berman J, Ben-Ami R. Candida haemulonii and Candida auris: emerging multidrug-resistant species with distinct virulence and epidemiological characteristics. Open Forum Infect Dis. 2016;3:124.

22. Sarma S, Upadhyay S. Current perspective on emergence, diagnosis and drug resistance in Candida auris. Infect Drug Resist. 2017;10:155-65.

23. Fakhim H, Vaezi A, Dannaoui E, Chowdhary A, Nasiry D, Faeli L, et al. Comparative virulence of Candida auris with Candida haemulonii, Candida glabrata and Candida albicans in a murine model. Mycoses. 2018;61:377-82.

24. Chowdhary A, Anil Kumar V, Sharma C, Prakash A, Agarwal K, Babu R, et al. Multidrug-resistant endemic clonal strain of Candida auris in India. Eur J Clin Microbiol Infect Dis. 2014;33:919-26.

25. Sharma C, Kumar N, Pandey R, Meis JF, Chowdhary A. Whole genome sequencing of emerging multidrug resistant Candida auris isolates in India demonstrates low genetic variation. New Microbes New Infect. 2016;13:77-82.

26. Jeffery-Smith A, Taori SK, Schelenz S, Jeffery K, Johnson EM, Borman A, et al. Candida auris: a review of the literature. Clin Microbiol Rev. 2018; 31:e00029-17.

27. Bougnoux M-E, Brun S, Zahar J-R. Healthcare-associated fungal outbreaks: new and uncommon species, new molecular tools for investigation and prevention. Antimicrob Resist Infect Control. 2018;7:45.

28. Kohlenberg A, Struelens MJ, Monnet DL, Plachouras D. Candida auris: epidemiological situation, laboratory capacity and preparedness in European Union and European Economic Area countries, 2013 to 2017. Euro Surveill. 2018;23:18-00136.

29. Borman AM, Szekely A, Johnson EM. Comparative pathogenicity of United Kingdom isolates of the emerging pathogen Candida auris and other key pathogenic Candida species. mSphere. 2016;1:e00189-16.
30. Larkin E, Hager C, Chandra J, Mukherjee PK, Retuerto M, Salem I, et al. The emerging pathogen Candida auris: growth phenotype, virulence factors, activity of antifungals, and effect of SCY-078, a novel glucan synthesis inhibitor, on growth morphology and biofilm formation. Antimicrob Agents Chemother. 2017;61:e02396-16.

31. Munoz JF, Gade L, Chow NA, Loparev VN, Juieng P, Farrer RA, et al. Genomic basis of multidrug-resistance, mating, and virulence in Candida auris and related emerging species. bioRxiv. 2018:299917. https://doi.org/10. $1101 / 299917$.

32. Girard V, Mailler S, Chetry M, Vidal C, Durand G, van Belkum A, et al. Identification and typing of the emerging pathogen Candida auris by matrix-assisted laser desorption ionisation time of flight mass spectrometry. Mycoses. 2016:59:535-8.

33. Mizusawa M, Miller H, Green R, Lee R, Durante M, Perkins R, et al. Can multidrug-resistant Candida auris be reliably identified in clinical microbiology laboratories? J Clin Microbiol. 2017;55:638-40.

34. Wattal C, Oberoi JK, Goel N, Raveendran R, Khanna S. Matrix-assisted laser desorption ionization time of flight mass spectrometry (MALDI-TOF MS) for rapid identification of micro-organisms in the routine clinical microbiology laboratory. Eur J Clin Microbiol Infect Dis. 2017;36:807-12.

35. Prakash A, Sharma C, Singh A, Kumar Singh P, Kumar A, Hagen F, et al. Evidence of genotypic diversity among Candida auris isolates by multilocus sequence typing, matrix-assisted laser desorption ionization time-of-flight mass spectrometry and amplified fragment length polymorphism. Clin Microbiol Infect. 2016;22:277.e1-9.

36. Kordalewska M, Zhao Y, Lockhart SR, Chowdhary A, Berrio I, Perlin DS. Rapid and accurate molecular identification of the emerging multidrug-resistant pathogen Candida auris. J Clin Microbiol. 2017:55:2445-52.

37. Leach L, Zhu Y, Chaturvedi S. Development and validation of a real-time PCR assay for rapid detection of Candida auris from surveillance samples. J Clin Microbiol. 2018;56:e01223-17.

38. Chowdhary A, Sharma C, Duggal S, Agarwal K, Prakash A, Singh PK, et al. New clonal strain of Candida auris, Delhi, India. Emerg Infect Dis. 2013;19: 1670-3.

39. Chatterjee S, Alampalli SV, Nageshan RK, Chettiar ST, Joshi S, Tatu US. Draft genome of a commonly misdiagnosed multidrug resistant pathogen Candida auris. BMC Genomics. 2015;16:686.

40. Lockhart SR, Berkow EL, Chow N, Welsh RM. Candida auris for the clinical microbiology laboratory: not your grandfather's Candida species. Clin Microbiol Newsl. 2017:39:99-103.

41. Lockhart SR, Etienne KA, Vallabhaneni S, Farooqi J, Chowdhary A, Govender $N P$, et al. Simultaneous emergence of multidrug-resistant Candida auris on 3 continents confirmed by whole-genome sequencing and epidemiological analyses. Clin Infect Dis. 2017;64:134-40.

42. Lee WG, Shin JH, Uh Y, Kang MG, Kim SH, Park KH, et al. First three reported cases of nosocomial fungemia caused by Candida auris. J Clin Microbiol. 2011:49:3139-42.

43. Oh BJ, Shin JH, Kim M-N, Sung H, Lee K, Joo MY, et al. Biofilm formation and genotyping of Candida haemulonii, Candida pseudohaemulonii, and a proposed new species (Candida auris) isolates from Korea. Med Mycol. 2011; 49:98-102.

44. Chakrabarti A, Sood P, Rudramurthy SM, Chen S, Kaur H, Capoor M, et al. Incidence, characteristics and outcome of ICU-acquired candidemia in India. Intensive Care Med. 2015;41:285-95.

45. Mohd Tap R, Lim TC, Kamarudin NA, Ginsapu SJ, Abd Razak MF, Ahmad N, et al. A fatal case of Candida auris and Candida tropicalis Candidemia in neutropenic patient. Mycopathologia. 2018;183:559-64.

46. Magobo RE, Corcoran C, Seetharam S, Govender N, Naicker S. Candida auris: an emerging, azole-resistant pathogen causing candidemia in South Africa. Int J Infect Dis. 2014;21:215. https://doi.org/10.1016/j.jij.2014.03.869.

47. Mohsin J, Hagen F, Al-Balushi ZAM, de Hoog GS, Chowdhary A, Meis JF, et al. The first cases of Candida auris candidaemia in Oman. Mycoses. 2017;60: 569-75.

48. Al-Siyabi T, Busaidi Al I, Balkhair A, Al-Muharrmi Z, Al-Salti M, Al'Adawi B. First report of Candida auris in Oman: clinical and microbiological description of five candidemia cases. J Inf Secur. 2017;75:373-6.

49. Okinda N, Kagotho E, Castanheira M, Njuguna A, Omuse G, Makau P. P0065 Candidemia at a referral hospital in sub-Saharan Africa: emergence of Candida auris as a major pathogen. 2014. 24 ${ }^{\text {th }}$ ECCMID.

50. Emara M, Ahmad S, Khan Z, Joseph L, Al-Obaid I, Purohit P, et al. Candida auris candidemia in Kuwait, 2014. Emerg Infect Dis. 2015;21:1091-2. 
51. Belkin A, Gazit Z, Keller N, Ben-Ami R, Wieder-Finesod A, Novikov A, et al. Candida auris infection leading to nosocomial transmission, Israel, 2017. Emerg Infect Dis. 2018;24:801.

52. Alatoom A, Sartawi M, Lawlor K, AbdelWareth L, Thomsen J, Nusair A, et al. Persistent candidemia despite appropriate fungal therapy: first case of Candida auris from the United Arab Emirates. Int J Infect Dis. 2018;70:36-7.

53. Abdalhamid B, Almaghrabi R, Althawadi S, Omrani A. First report of Candida auris infections from Saudi Arabia. J Infect Public Health. 2018;11:598-9.

54. Wang X, Bing J, Zheng Q, Zhang F, Liu J, Yue H, et al. The first isolate of Candida auris in China: clinical and biological aspects. Emerg Microbes Infect. 2018;7:93.

55. Parra-Giraldo CM, Valderrama SL, Cortes-Fraile G, Garzon JR, Ariza BE, Morio F, et al. First report of sporadic cases of Candida auris in Colombia. Int J Infect Dis. 2018;69:63-7.

56. Morales-Lopez SE, Parra-Giraldo CM, Ceballos-Garzon A, Martinez HP Rodriguez GJ, Alvarez-Moreno CA, et al. Invasive infections with multidrugresistant yeast Candida auris, Colombia. Emerg Infect Dis. 2017:23:162-4.

57. Escandón P. Notes from the field: surveillance for Candida auris-Colombia, September 2016-May 2017. MMWR Morb Mortal Wkly Rep. 2018;67:459-60.

58. Calvo B, Melo ASA, Perozo-Mena A, Hernandez M, Francisco EC, Hagen F, et al. First report of Candida auris in America: clinical and microbiological aspects of 18 episodes of candidemia. J Inf Secur. 2016;73:369-74.

59. McCarthy M. Hospital transmitted Candida auris infections confirmed in the US. BMJ. 2016;355:15978

60. Vallabhaneni S, Kallen A, Tsay S, Chow N, Welsh R, Kerins J, et al. Investigation of the first seven reported cases of Candida auris, a globally emerging invasive, multidrug-resistant fungus-United States, May 2013August 2016. Am J Transplant. 2017;17:296-9.

61. Tsay S, Welsh RM, Adams EH, Chow NA, Gade L, Berkow EL, et al. Notes from the field: ongoing transmission of Candida auris in health care facilities - United States, June 2016-May 2017. MMWR Morb Mortal Wkly Rep. 2017; 66:514-5.

62. Vasilyeva N, Kruglov A, Pchelin I, Riabinin I, Raush E, Chilina G, et al. P0311 the first Russian case of candidaemia due to Candida auris. 2018. 28th ECCMID.

63. Schwartz IS, Hammond GW. First reported case of multidrug-resistant Candida auris in Canada. Can Commun Dis Rep. 2017:43:150.

64. Ramos R, Caceres DH, Perez M, Garcia N, Castillo W, Santiago E, et al. Emerging multidrug-resistant Candida duobushaemulonii infections in Panama hospitals: importance of laboratory surveillance and accurate identification. J Clin Microbiol. 2018;56:e00371-18.

65. Arauz AB, Caceres DH, Santiago E, Armstrong P, Arosemena S, Ramos C, et al. Isolation of Candida auris from 9 patients in Central America: importance of accurate diagnosis and susceptibility testing. Mycoses. 2018;61:44-7.

66. Schelenz S, Hagen F, Rhodes JL, Abdolrasouli A, Chowdhary A, Hall A, et al. First hospital outbreak of the globally emerging Candida auris in a European hospital. Antimicrob Resist Infect Control. 2016:5:35.

67. Ruiz Gaitan AC, Moret A, Lopez Hontangas JL, Molina JM, Aleixandre Lopez $\mathrm{Al}$, Cabezas AH, et al. Nosocomial fungemia by Candida auris: first four reported cases in continental Europe. Rev Iberoam Micol. 2017;34:23-7.

68. Riat A, Neofytos D, Coste A, Harbarth S, Bizzini A, Grandbastien B, et al. First case of Candida auris in Switzerland: discussion about preventive strategies. Swiss Med Wkly. 2018;148:w14622.

69. Ruiz-Gaitan A, Moret AM, Tasias-Pitarch M, Aleixandre-Lopez Al, MartinezMorel H, Calabuig E, et al. An outbreak due to Candida auris with prolonged colonisation and candidaemia in a tertiary care European hospital. Mycoses. 2018;61:498-505.

70. https://ecdc.europa.eu/en/publications-data/rapid-risk-assessment-candidaauris-healthcare-settings-europe. Accessed 22 July 2018.

71. https://www.cdc.gov/fungal/diseases/candidiasis/c-auris-alert-09-17.html. Accessed 22 July 2018.

72. https://www.cdc.gov/fungal/diseases/candidiasis/candida-auris-alert.html Accessed 22 July 2018

73. Borman AM, Szekely A, Johnson EM. Isolates of the emerging pathogen Candida auris present in the UK have several geographic origins. Med Mycol. 2017:55:563-7.

74. Kumar D, Banerjee T, Pratap CB, Tilak R. Itraconazole-resistant Candida auris with phospholipase, proteinase and hemolysin activity from a case of vulvovaginitis. J Infect Dev Ctries. 2015;9:435-7.

75. Azar MM, Turbett SE, Fishman JA, Pierce VM. Donor-derived transmission of Candida auris during lung transplantation. Clin Infect Dis. 2017;65:1040-2.
76. Navalkele BD, Revankar S, Chandrasekar P. Candida auris: a worrisome, globally emerging pathogen. Expert Rev Anti-Infect Ther. 2017;15:819-27.

77. Warris A. Candida auris, what do paediatricians need to know? Arch Dis Child. 2018. https://doi.org/10.1136/ archdischild-2017-313960.

78. https://www.gov.uk/government/publications/candida-auris-laboratoryinvestigation-management-and-infection-prevention-and-control. Accessed 16 July 2018.

79. https://www.cdc.gov/fungal/candida-auris/recommendations.html. Accessed 22 July 2018.

80. http://www.nicd.ac.za/index.php/interim-guidance-for-the-management-ofcandida-auris-infections-in-south-african-hospitals/. Accessed 22 July 2018.

81. Biswal M, Rudramurthy SM, Jain N, Shamanth AS, Sharma D, Jain K, et al. Controlling a possible outbreak of Candida auris infection: lessons learnt from multiple interventions. J Hosp Infect. 2017;97:363-70.

82. Eyre D, Sheppard A, Madder H, Moir I, Moroney R, Quan TP, et al. 00172 Epidemiology and successful control of a Candida auris outbreak in a UK intensive care unit driven by multi-use patient monitoring equipment. 2018. 28th ECCMID.

83. Escandón P, Chow NA, Caceres DH, Gade L, Berkow EL, Armstrong P, et al. Molecular epidemiology of Candida auris in Colombia reveals a highlyrelated, country-wide colonization with regional patterns in amphotericin B resistance. Clin Infect Dis. 2018. https://doi.org/10.1093/cid/ciy411.

84. Welsh RM, Bentz ML, Shams A, Houston H, Lyons A, Rose LJ, et al. Survival, persistence, and isolation of the emerging multidrug-resistant pathogenic yeast Candida auris on a plastic health care surface. J Clin Microbiol. 2017; 55:2996-3005

85. Cadnum JL, Shaikh AA, Piedrahita CT, Sankar T, Jencson AL, Larkin EL, et al. Effectiveness of disinfectants against Candida auris and other Candida species. Infect Control Hosp Epidemiol. 2017;38:1240-3.

86. Ku TSN, Walraven CJ, Lee SA. Candida auris: disinfectants and implications for infection control. Front Microbiol. 2018;9:726.

87. Sherry L, Ramage G, Kean R, Borman A, Johnson EM, Richardson MD, et al. Biofilm-forming capability of highly virulent, multidrug-resistant Candida auris. Emerg Infect Dis. 2017;23:328-31.

88. Kean R, Sherry L, Townsend E, McKloud E, Short B, Akinbobola A, et al. Surface disinfection challenges for Candida auris: an in-vitro study. J Hosp Infect. 2018;98:433-6.

89. Kean R, MCKloud E, Townsend EM, Sherry L, Delaney C, Jones BL, et al. The comparative efficacy of antiseptics against Candida auris biofilms. Int J Antimicrob Agents. 2018. https://doi.org/10.1016/j.ijantimicag.2018.05.007.

90. Chowdhary A, Prakash A, Sharma C, Kordalewska M, Kumar A, Sarma S, et al. A multicentre study of antifungal susceptibility patterns among 350 Candida auris isolates (2009-17) in India: role of the ERG11 and FKS1 genes in azole and echinocandin resistance. J Antimicrob Chemother. 2018;73:891-9.

91. Mathur P, Hasan F, Singh PK, Malhotra R, Walia K, Chowdhary A. Five-year profile of candidemia at an Indian trauma center: high rates of Candida auris blood stream infections. Mycoses. 2018. https://doi.org/10.1111/myc.12790.

92. Morio F, Loge C, Besse B, Hennequin C, Le Pape P. Screening for amino acid substitutions in the Candida albicans Erg11 protein of azole-susceptible and azole-resistant clinical isolates: new substitutions and a review of the literature. Diagn Microbiol Infect Dis. 2010;66:373-84.

93. Xiang M-J, Liu J-Y, Ni P-H, Wang S, Shi C, Wei B, et al. Erg11 mutations associated with azole resistance in clinical isolates of Candida albicans. FEMS Yeast Res. 2013;13:386-93.

94. Cowen LE, Sanglard D, Howard SJ, Rogers PD, Perlin DS. Mechanisms of antifungal drug resistance. Cold Spring Harb Perspect Med. 2014;5:a019752.

95. Rhodes J, Abdolrasouli A, Farrer RA, Cuomo CA, Aanensen DM, ArmstrongJames D, et al. Genomic epidemiology of the UK outbreak of the emerging human fungal pathogen Candida auris. Emerg Microbes Infect. 2018;7:43.

96. Kordalewska M, Lee A, Park S, Berrio I, Chowdhary A, Zhao Y, et al. Understanding echinocandin resistance in the emerging pathogen Candida auris. Antimicrob Agents Chemother. 2018;62:e00238-18.

97. Berkow EL, Angulo D, Lockhart SR. In vitro activity of a novel glucan synthase inhibitor, SCY-078, against clinical isolates of Candida auris. Antimicrob Agents Chemother. 2017;61:e00435-17.

98. Basso V, Garcia A, Tran DQ, Schaal JB, Tran P, Ngole D, et al. Fungicidal potency and mechanisms of theta-Defensins against multidrug-resistant Candida species. Antimicrob Agents Chemother. 2018:62:e00111-8.

99. Hager CL, Larkin EL, Long L, Zohra Abidi F, Shaw KJ, Ghannoum MA. In vitro and in vivo evaluation of the antifungal activity of APX001A/APX001 against Candida auris. Antimicrob Agents Chemother. 2018;62:e02319-7. 
100. Zhao M, Lepak AJ, VanScoy B, Bader JC, Marchillo K, Vanhecker J, et al. In vivo pharmacokinetics and pharmacodynamics of APX001 against Candida in a neutropenic disseminated candidiasis mouse model. Antimicrob Agents Chemother. 2018;62:e02542-17.

101. Berkow EL, Lockhart SR. Activity of CD101, a long-acting echinocandin, against clinical isolates of Candida auris. Diagn Microbiol Infect Dis. 2018;90:196-7.

Ready to submit your research? Choose BMC and benefit from:

- fast, convenient online submission

- thorough peer review by experienced researchers in your field

- rapid publication on acceptance

- support for research data, including large and complex data types

- gold Open Access which fosters wider collaboration and increased citations

- maximum visibility for your research: over $100 \mathrm{M}$ website views per year

At $\mathrm{BMC}$, research is always in progress.

Learn more biomedcentral.com/submissions 